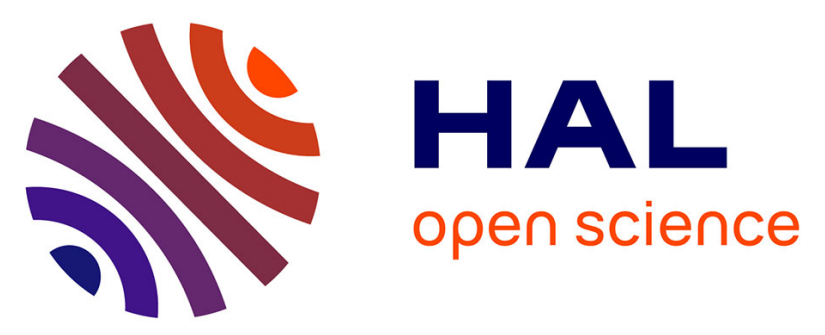

\title{
Enhancement of the Dzyaloshinskii-Moriya interaction and domain wall velocity through interface intermixing in $\mathrm{Ta} / \mathrm{CoFeB} / \mathrm{MgO}$
}

L Herrera Diez, M. Voto, A. Casiraghi, M. Belmeguenai, Y. Roussigne, G. Durin, A. Lamperti, R. Mantovan, V. Sluka, V. Jeudy, et al.

\section{To cite this version:}

L Herrera Diez, M. Voto, A. Casiraghi, M. Belmeguenai, Y. Roussigne, et al.. Enhancement of the Dzyaloshinskii-Moriya interaction and domain wall velocity through interface intermixing in $\mathrm{Ta} / \mathrm{CoFeB} / \mathrm{MgO}$. Physical Review B: Condensed Matter and Materials Physics (1998-2015), 2019, 99 (5), 10.1103/PhysRevB.99.054431 . hal-02331239

\section{HAL Id: hal-02331239 \\ https://hal.science/hal-02331239}

Submitted on 24 Oct 2019

HAL is a multi-disciplinary open access archive for the deposit and dissemination of scientific research documents, whether they are published or not. The documents may come from teaching and research institutions in France or abroad, or from public or private research centers.
L'archive ouverte pluridisciplinaire HAL, est destinée au dépôt et à la diffusion de documents scientifiques de niveau recherche, publiés ou non, émanant des établissements d'enseignement et de recherche français ou étrangers, des laboratoires publics ou privés. 


\title{
Enhancement of Dzyaloshinskii-Moriya interaction and domain wall velocity through interface intermixing in $\mathrm{Ta} / \mathrm{CoFeB} / \mathrm{MgO}$
}

\author{
L. Herrera Diez, ${ }^{1, *}$ M. Voto,${ }^{2}$ A. Casiraghi,${ }^{3}$ M. Belmeguenai, ${ }^{4}$ Y. Roussigné,${ }^{4}$ \\ G. Durin, ${ }^{3}$ A. Lamperti, ${ }^{5}$ R. Mantovan, ${ }^{5}$ V. Sluka, ${ }^{6}$ V. Jeudy,${ }^{7}$ Y. T. Liu, ${ }^{1}$ A. \\ Stashkevich, ${ }^{4}$ S. M. Chérif, ${ }^{4}$ J. Langer ${ }^{8}$ B. Ocker ${ }^{8}$ L. Lopez-Diaz, ${ }^{2}$ and D. Ravelosona ${ }^{1,9}$ \\ ${ }^{1}$ Centre de Nanosciences et de Nanotechnologies, CNRS, Univ. Paris-Sud, \\ Université Paris-Saclay, C2N Orsay, 91405 Orsay cedex, France \\ ${ }^{2}$ Departamento de Física Aplicada, Universidad de Salamanca, \\ Plaza de la Merced $s / n .37008$ Salamanca, Spain \\ ${ }^{3}$ Istituto Nazionale di Ricerca Metrologica, Strada delle Cacce 91, 10135 Torino, Italy. \\ ${ }^{4}$ Laboratoire des Sciences des Procédés et des Matériaux, CNRS-UPR 3407, \\ Université Paris 13, Sorbonne Paris Cité, 93430 Villetaneuse, France. \\ ${ }^{5}$ CNR-IMM, Unit of Agrate Brianza, Via C. Olivetti 2, Agrate Brianza, MB, Italy \\ ${ }^{6}$ Department of Physics, New York University, New York, New York 10003, USA. \\ ${ }^{7}$ Laboratoire de Physique des Solides, CNRS, Univ. Paris-Sud, \\ Université Paris-Saclay, 91405 Orsay Cedex, France \\ ${ }^{8}$ Singulus Technology AG, Hanauer Landstrasse 103, 63796 Kahl am Main, Germany. \\ ${ }^{9}$ Spin-Ion technologies, C2N, Avenue de la Vauve, 91120 Palaiseau, FRANCE
}

(Dated: September 10, 2019)

\begin{abstract}
The interfacial Dzyaloshinskii-Moriya Interaction (DMI) plays a crucial role in chiral domain wall (DW) motion, favoring fast DW velocities. We explore the effect of interface disorder on DMI and DW dynamics in perpendicular magnetized $\mathrm{Ta} / \mathrm{CoFeB} / \mathrm{MgO}$ thin films. Light $\mathrm{He}^{+}$irradiation has been used to gently engineer interface intermixing on a scale of $0.1 \mathrm{~nm}$. We demonstrate that a slight modification of the $\mathrm{Ta} / \mathrm{CoFeB}$ interface leads to an increase of the DMI value accompanied by an enhancement of DW velocity in the flow regime. Using micromagnetic simulations based on granular structures, we show that the enhancement of DW velocity is mainly related to an increase in the distribution of magnetic parameters related to the interface. We further infer that the DMI modulation is related to the asymmetric disorder induced by irradiation leading to alloying with the Ta buffer layer. Understanding the role of disorder is therefore crucial for the design of future devices where post-growth interface alloying can be used to finely tune the DMI.
\end{abstract}

PACS numbers: 75.30.Gw, 75.60.Ch, 75.70.-i, 75.78.Fg

Keywords: $\mathrm{CoFeB} / \mathrm{MgO}$, perpendicular magnetic anisotropy, domain wall motion, creep law

\section{INTRODUCTION}

Interface engineering applied to ultra-thin magnetic films has emerged as a determining path for the development of low power spintronics technologies. In particular, the Dzyaloshinskii-Moriya interaction $(\mathrm{DMI})^{1-4}$, which is intimately related to the breaking of the symmetry at the interfaces of the magnetic film, is of major importance. The magnitude and sign of the DMI can be highly dependent on the interaction of the magnetic film with a neighbouring metal with high spin orbit coupling ${ }^{5,6}$ and its ratio to the magnetization value can be the key to high DW velocities ${ }^{7}$. DMI is also responsible for the appearance of chiral DWs $\mathrm{s}^{4,8}$ and skyrmions ${ }^{9}$, topologically protected structures that can be manipulated by using electrical currents ${ }^{10}$ and spin-orbit torques ${ }^{11}$ and therefore hold great potential for a new generation of spintronics technologies.

In this context, one important limitation for the motion of solitonic magnetic objects is the influence of structural inhomogeneities such as interface intermixing and roughness or grain textures. Pinning due to defects leads to the thermally activated creep regime of DW motion at low drive, which has been extensively studied for DWs in a variety of materials and structures ${ }^{12-16}$. Along this line, the full extent of the impact of disorder on the DMI value is a key aspect. For instance, in symmetric epitaxial $\mathrm{Pt} / \mathrm{Co} / \mathrm{Pt}$ structures DMI can still appear due to a difference between the quality of the Co interfaces ${ }^{17,18}$ In the same material, density functional calculations indicate that DMI could be significantly reduced upon dusting the interface with a third element ${ }^{19}$. Additionally, the DMI value has been shown to depend on the degree of hybridization between the $3 d$ orbitals of the ferromagnetic metal and the $5 d$ orbitals of the heavy metal highlighting the importance of alloying at the interface ${ }^{6}$.

In this paper, we investigate the impact of interface intermixing in $\mathrm{Ta} / \mathrm{CoFeB} / \mathrm{MgO}$ materials on the DMI by using $\mathrm{He}^{+}$ion irradiation to gently tune the interface morphology at the atomic scale. DMI is found to increase as a function of the irradiation dose in films where DMI is nearly quenched in the pristine state. This increase in DMI is accompanied by an increase in DW velocities in the flow regime. Our results show that the DMI and the maximum DW velocities are significantly dependent on interfacial alloying. 


\section{DW MOTION FROM THE CREEP INTO THE FLOW REGIME}

The samples investigated are $\mathrm{Si} / \mathrm{SiO}_{2} / \mathrm{Ta}(5$ $\mathrm{nm}) / \mathrm{Co}_{20} \mathrm{Fe}_{60} \mathrm{~B}_{20}(1 \mathrm{~nm}) / \mathrm{MgO}(2 \mathrm{~nm}) / \mathrm{Ta}(3 \mathrm{~nm})$ films annealed at $300^{\circ} \mathrm{C}$ exhibiting perpendicular magnetic anisotropy (PMA). The films were subsequently irradiated at doses (ID) up to $5.5 \times 10^{19} \mathrm{He}^{+} / \mathrm{m}^{2}$. Fig. 1 (a) shows a sketch of the sample structure.

DW motion in $\mathrm{He}^{+}$irradiated $\mathrm{CoFeB}$ films has been thoroughly studied in the thermally activated, and highly defect-sensitive, creep regime of DW motion ${ }^{16}$. These investigations were centered around the effects of the modification of the $\mathrm{CoFeB} / \mathrm{MgO}$ interface. A depletion of the $\mathrm{Fe}$ content at this interface is found upon increasing the irradiation dose which is in line with the observed reduction in magnetisation $\left(M_{s}\right)$ and the effective anisotropy constant $\left(K_{\text {eff }}\right)^{16,20}$. For the present study the dependence of the anisotropy field $H_{K}=2 K_{\text {eff }} / M_{s}$ is given in Fig. 1. (b). The end of the highly defect sensitive creep regime of DW motion is marked by the depinning field $H_{d e p}{ }^{12-14}$ which has been found to increase monotonically with irradiation dose ${ }^{16}$. Fig. 1 (c) (blue circles, left axis) shows this increase in $H_{d e p}$ which has been calculated by evaluating the intersection between the creep and depinning regimes of DW motion ${ }^{21}$. As previously reported ${ }^{16}$, the irradiation treatment widens the magnetic field range in which the creep regime can be observed implying an increased interaction with defects for higher IDs leading to an increase in $H_{\text {dep }}$. However, the value of the DW velocity at $H_{d e p}$ does not reflect this trend, $v\left(H_{d e p}\right)$ (Fig 1 (c), cyan circles, right axis) shows an initial increase up to $\mathrm{ID}=8 \times 10^{18} \mathrm{He}^{+} / \mathrm{m}^{2}$ followed by a monotonic decrease. This feature has been found to be an early indication of the dynamics at higher fields, well outside of the creep regime. The full DW velocity plot for pristine and irradiated films with IDs ranging from $1 \times 10^{18}$ to $1.6 \mathrm{x}$ $10^{19} \mathrm{He}^{+} / \mathrm{m}^{2}$ is shown in Fig. 2 (a). The DW velocities in a field range of 53-56 $\mathrm{mT}$ (see the highlighted bar in Fig. 2 (a)) are plotted in Fig 2 (b) as a function of ID showing the trend in the maximum velocity as a function of ID. The dependence of the maximum DW velocity on ID resembles that of $v\left(H_{d e p}\right)$ with an initial increase up to $\mathrm{ID}=8 \times 10^{18} \mathrm{He}^{+} / \mathrm{m}^{2}$ followed by a reduction at higher IDs. It is interesting to notice that the end of the creep regime can provide qualitative information about the behaviour of the maximum velocities well beyond the depinning regime though $v\left(H_{d e p}\right)$ but not through $H_{\text {dep }}$, which shows a monotonic increase that reflects the behaviour of the DW velocity deep into the creep regime.

It is important to mention that the velocity curves beyond the exponential creep regime do not show the linear dependence predicted by the so-called 'one dimensional model' of DW motion that describes a one dimensional interface (DW) propagating in a two dimensional medium $(\text { film })^{12}$. The DW velocity leaves a)

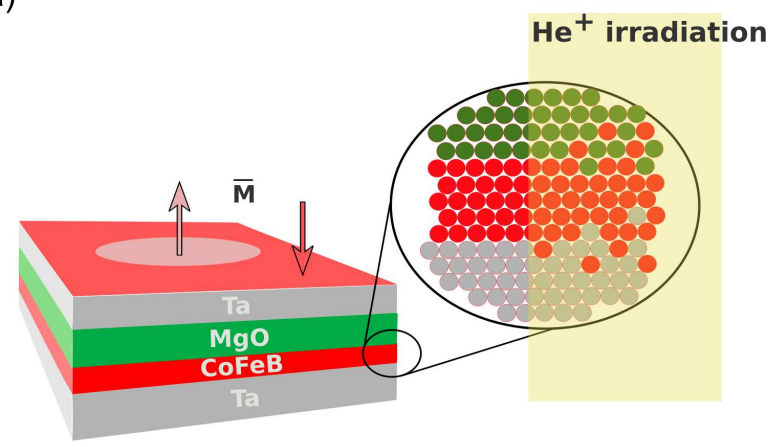

b)

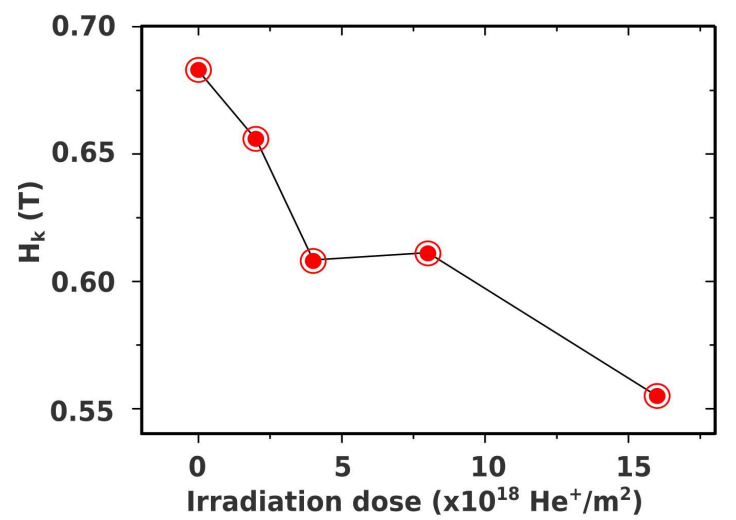

c)

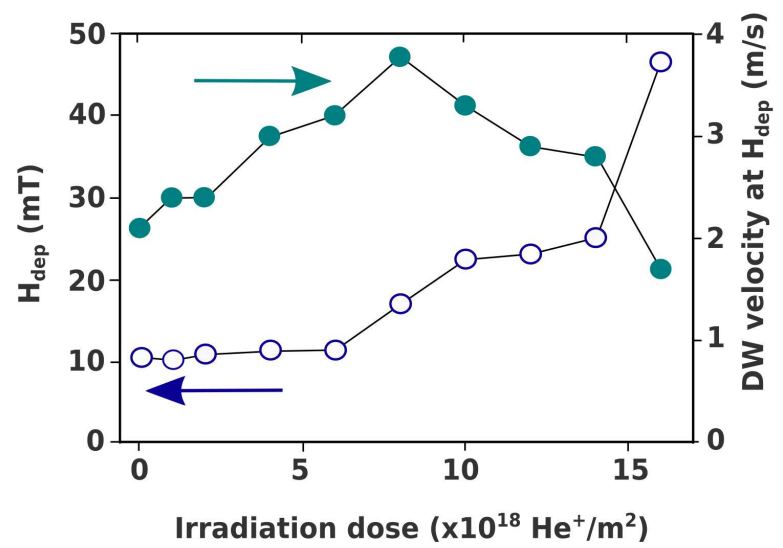

FIG. 1: (a) Sketch depicting the perpendicularly magnetised $\mathrm{CoFeB}$ stack and the effect of $\mathrm{He}^{+}$irradiation at the interfaces. (b) Anisotropy field $H_{k}$ as a function of the irradiation dose. (c) Depinning field $H_{\text {dep }}$ (blue circles, left axis) and its corresponding DW velocity $v\left(H_{d e p}\right)$ (cyan circles, right axis) as a function of irradiation dose.

the exponential creep regime to transition (depinning regime) into a final saturation velocity regime where DW velocity remains fairly unchanged over a wide range of magnetic fields. This DW velocity profile has already been observed ${ }^{7,22}$ and it has been attributed to the system being locked in the velocity plateau in the high 
(a)

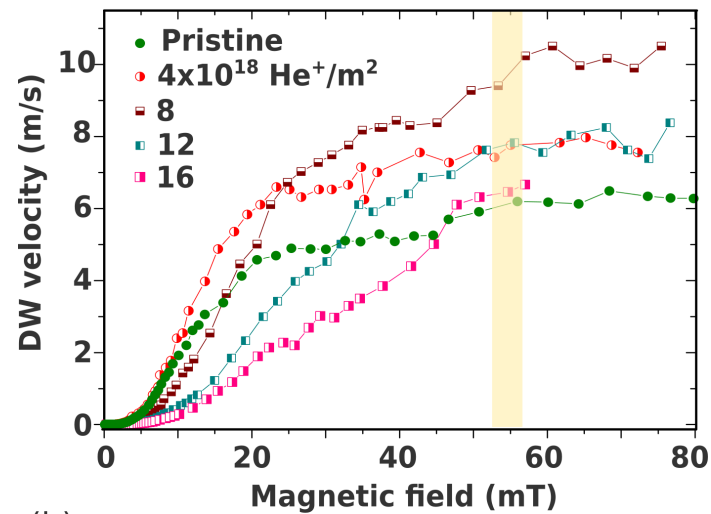

(b)

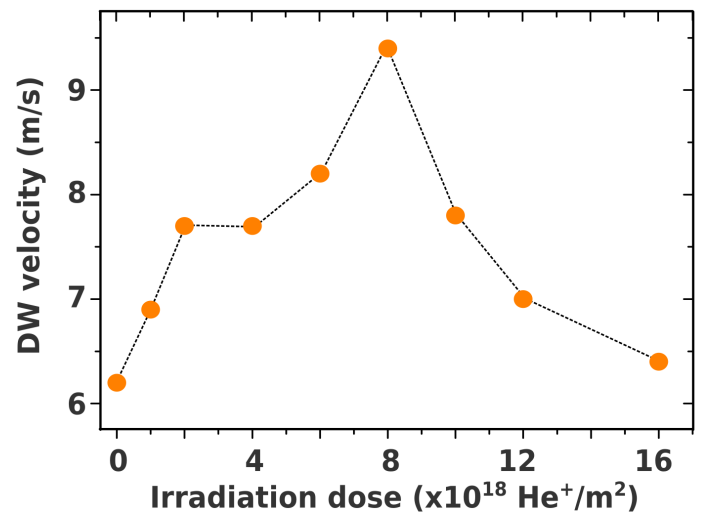

FIG. 2: (a) DW velocity as a function of the applied magnetic field for pristine and irradiated films. (b) DW velocity in the range 53-56 $\mathrm{mT}$ as a function of the irradiation dose. The yellow bar in (a) shows the velocity region that is plotted in (b) in order to illustrate the trend in the maximum velocity vs. ID.

field side of the Walker breakdown $H_{W}$, the transition point between the steady and precessional flow regimes. In equivalent $\mathrm{CoFeB}$ materials $H_{W}$ has been calculated to appear at very low fields and well into the creep regime $^{23}$, this determines that the system is already beyond the steady flow regime when it moves out of the creep regime and, as mentioned, it is trapped in a velocity plateau from which the system could break free at high enough fields ${ }^{22}$.

The observed increase in $H_{d e p}$ is compatible with an increase in the level of disorder in the system ${ }^{16}$. In order to complete this analysis the irradiation-induced changes in the structure and properties of the bottom $\mathrm{Ta} / \mathrm{CoFeB}$ interface were investigated. As mentioned, the structure of this interface is of critical relevance to define the role of the DMI interaction in pristine/irradiated systems. Fig. 3 (a) shows specular X-Ray reflectivity performed using $\mathrm{Cu} \mathrm{K}-\alpha$ X-rays with wavelength $\lambda=0.154 \mathrm{~nm}$ for a pristine film and a film irradiated at $\mathrm{ID}=5 \mathrm{x}$ $10^{18} \mathrm{He}^{+} / \mathrm{m}^{2}$. Fitting using the software MAUD ${ }^{24}$ (solid lines) allows for the extraction of the values of the interface widths considering both the topological (a)

(b)
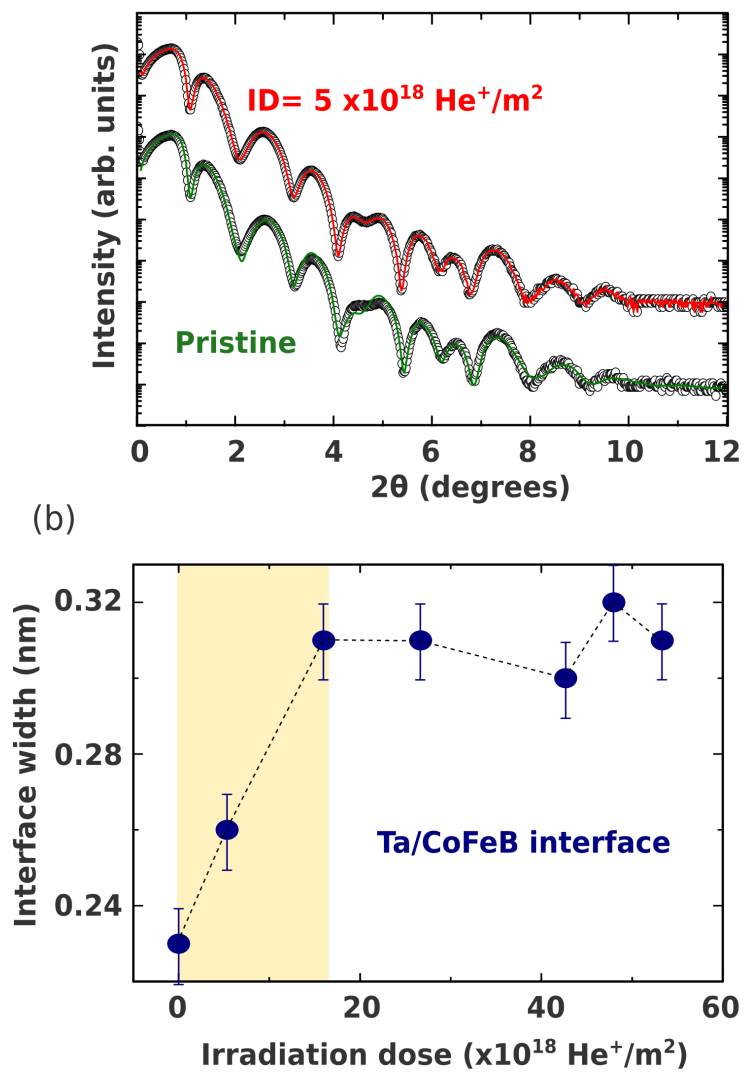

FIG. 3: (a) X-ray reflectivity profiles for a pristine and an irradiated films at $\mathrm{ID}=5 \times 10^{18} \mathrm{He}^{+} / \mathrm{m}^{2}$, the corresponding model is plotted as solid lines. (b) Width of the $\mathrm{Ta} / \mathrm{CoFeB}$ interface as a function of the irradiation dose.

roughness and chemical grading as a function of the irradiation dose. The extracted interface width is shown in Fig. 3 (b) for irradiation doses going up to 5.5 $\mathrm{x} 10^{19} \mathrm{He}^{+} / \mathrm{m}^{2}$. The initial value of $0.23 \pm 0.01 \mathrm{~nm}$ for the pristine material rapidly grows with ID up to about $\mathrm{ID}=1.5 \times 10^{19} \mathrm{He}^{+} / \mathrm{m}^{2}$, which corresponds to the range of irradiation doses where DW dynamics has been analysed. After this marked increase the width of the interface seems to stabilise at values between $0.31 \pm 0.01$ and $0.32 \pm 0.01 \mathrm{~nm}$, even when further irradiation at significantly higher doses is performed. This variation shows that irradiation has an important impact in the $\mathrm{Ta} / \mathrm{CoFeB}$ interface but also indicates that the irradiation-induced disorder might reach a saturation point within the ID range where DW motion was investigated.

Knowing that the width of the $\mathrm{Ta} / \mathrm{CoFeB}$ interfaces is modified by irradiation, an evaluation of the effect of irradiation on DMI has been conducted. This has been done by analysing magnetic domain wall motion in the presence of perpendicular and in-plane magnetic fields and also by analysing the surface spin-wave propagation asymmetry by Brillouin light spectroscopy. 


\section{IRRADIATION INDUCED DMI}

As presented by numerous studies in the literature, the presence of DMI can be evidenced in DW motion due to the changes induced in the internal structure of the DW, typically a Bloch to Néel type DW transition as a function of the strength of DMI and the appearance of a distinctive DW chirality depending on the sign of $\mathrm{DMI}^{8,25}$. In this context, the simultaneous application of an out-of plane magnetic field (to induce DW motion) and an in-plane field (to break the symmetry of the radial effective field induced by DMI) to a circular bubble domain induces an asymmetric expansion of the domain along the axis of the applied in-plane field, which can be related to the strength and sign of the $\mathrm{DMI}^{25,26}$. The measurements presented in Fig. 4 have been performed in the creep regime of DW motion using a perpendicular magnetic field of $1.9 \mathrm{mT}$ while sweeping an in-plane magnetic field. Fig. 4 shows the DW velocity as a function of the applied in-plane field for the right (open circles) and left (empty circles) propagating sides of the bubble domain. Each pair of velocity curves shows a minimum that directly corresponds to the field compensating the DMI effective field $H_{D M I}=\frac{D}{\mu_{0} M_{s} \delta}$ where $\delta=\sqrt{A / K_{\text {eff }}}$ is the domain wall width, $A$ is the exchange stiffness constant and $K_{\text {eff }}$ is the effective magnetic anisotropy ${ }^{25,26}$. Fig. 4 also shows Kerr microscopy images taken at $H_{x}=0$ and $\pm H_{x} \approx H_{D M I}$ where an asymmetry in the domain expansion is evidenced. The values of $D$ obtained by this method $\left(D_{D W}\right)$ are plotted in Fig. 5 (a, full circles) as a function of the irradiation dose. The values of $K_{\text {eff }}$ and $M_{s}$ used to calculate $D$ correspond to those informed in a previous report ${ }^{16}$ and $A$ has been fixed to $2.3 \times 10^{-11} \mathrm{~J} / \mathrm{m}$ for the non irradiated film based on an estimation done taking into account the values for pure Fe and $\mathrm{Co}$, the composition of the film and values reported in the literature ${ }^{27}$. The values of $A$ used for the irradiated films have been estimated by considering a weak dependence on $M_{s}^{2}$ in line with predictions and experimental observations ${ }^{28,29}$. This weak variation is also comparable to the $M_{s}$ dependence reported for thick $\mathrm{CoFeB}$ films ${ }^{30,31}$

Another common method of estimating the values of $D$ is to measure the DMI induced non-reciprocity of surface spin wave propagation ${ }^{26,32}$ by Brillouin light sprectroscopy (BLS). In BLS, inelastic scattering occurs between the incident light beam and spin waves producing a shift in the frequency of the light that can be due to an energy loss (spin wave creation) or an energy gain (spin wave absorption) which is evidenced as the so-called Stokes and anti-Stokes peaks, respectively, in an intensity vs. frequency plot. The presence of DMI shows as a non-reciprocity in the frequency values of the Stokes $\left(f_{S}\right)$ and anti-Stokes peaks $\left(f_{A S}\right)$ where the frequency difference $\Delta f=f_{S}-f_{A S}$ has the following linear dependence on the spin wave vector $k_{s w}$ :

$$
\Delta f=\frac{2 \gamma}{\pi M_{s}} D k_{s w}
$$

where $D$ is the DMI constant and $\gamma$ the giromagnetic constant. This linear dependence has been verified experimentally and it is extensively used to extract values of $D$ in a number of perpendicularly magnetised ferromagnetic thin films ${ }^{26,32}$. Fig. 5 (b) shows the dependence of the frequency shift $\Delta f$ on the wavevector of the spin waves $k_{s w}$ for the pristine material and films irradiated at $\mathrm{ID}=4,8,12$ and $16 \times 10^{18} \mathrm{He}^{+} / \mathrm{m}^{2}$. The profile obtained presents a linear dependence for intermediate IDs and high $k_{s w}$ that allows for the determination of the strength of the DMI by fitting to Eq. (1) and examining the slope of the curve. The result of this fitting is presented as empty squares in Fig. 5 (a).

A more complex scenario is present at low $k_{s w}$ values where $\Delta f$ tends to zero at $k_{s w}=0$ but not following the same linear dependence seen at higher $k_{s w}$. Moreover, a non zero intercept can be extrapolated from the linear dependence present at high $k_{s w}$ for all samples. This intercept is not considered by the model described by Eq. (1) and is thought to be related to an inhomogeneous component arising due to disorder. A detailed discussion on the dependence of the BLS signal on disorder will be presented in the last section of this manuscript.

The $D$ values obtained by considering the slope at high $k_{s w}$ are in the range of those obtained by DW motion experiments and follow a similar trend. It is worth noting that the values of $D$ obtained from DW motion experiments and BLS can often exhibit a certain degree of discrepancy due to the different scales in which these two techniques probe the sample. DW motion may capture a rather localised picture (over tens of square microns) while BLS can provide an average value over a larger area (about a milimeter square) ${ }^{26}$. Therefore, potential variations of $D$ within these length scales could be responsible for the discrepancy observed between the two methods. In addition, the significant inhomogeneous contribution present in the BLS signal in this system introduces a larger error bar. Nevertheless, both $D_{D W}$ and $D_{B L S}$ indicate that an increase in the magnitude of DMI can be produced with irradiation doses up to $\mathrm{ID}=8 \times 10^{18} \mathrm{He}^{+} / \mathrm{m}^{2}$. This is in close resemblance with the effects on the width of the Ta/CoFeB interface presented in Fig. 3 (b), which leads to the conclusion that a mechanism involving a large DMI sensitivity to the morphology of this interface is at play.

$\mathrm{He}^{+}$irradiation at energies of a few tens of $\mathrm{keV}$ limits recoils to about one atomic distance and the number of displaced atoms in the layers is of about $1 \times 10^{-2} / \mathrm{He}^{+} / \mathrm{nm}$ with all $\mathrm{He}^{+}$ions being implanted in the substrate ${ }^{33,34}$. In these conditions, $\mathrm{He}^{+}$irradiation allows for the exploration of non-equilibrium paths towards lower energy structural and chemical configurations. The $\mathrm{CoFeB} / \mathrm{MgO}$ interface is known to be more robust than the $\mathrm{Ta} / \mathrm{CoFeB}$ interface against mixing, since the mixing enthalpy favours the diffusion of $\mathrm{Fe}, \mathrm{Co}$ and $\mathrm{B}$ into Ta rather than into $\mathrm{MgO}^{35}$. As a result, a difference in the rate of intermixing between the bottom 
(a)
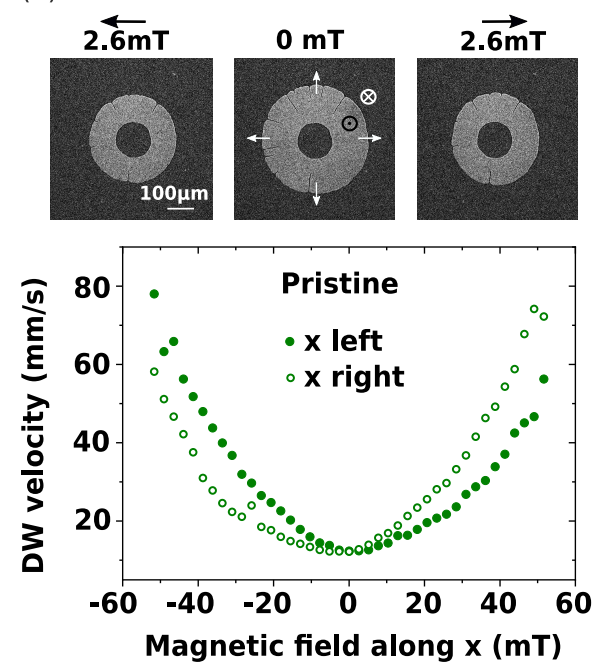

(c)
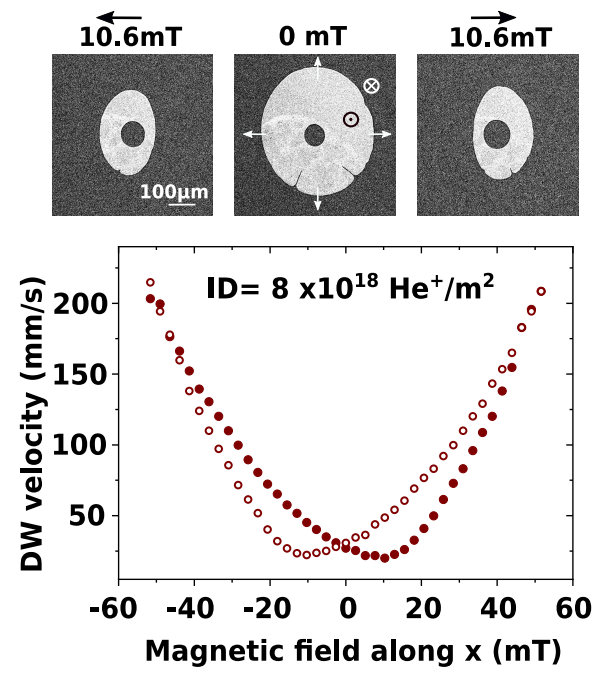

(b)
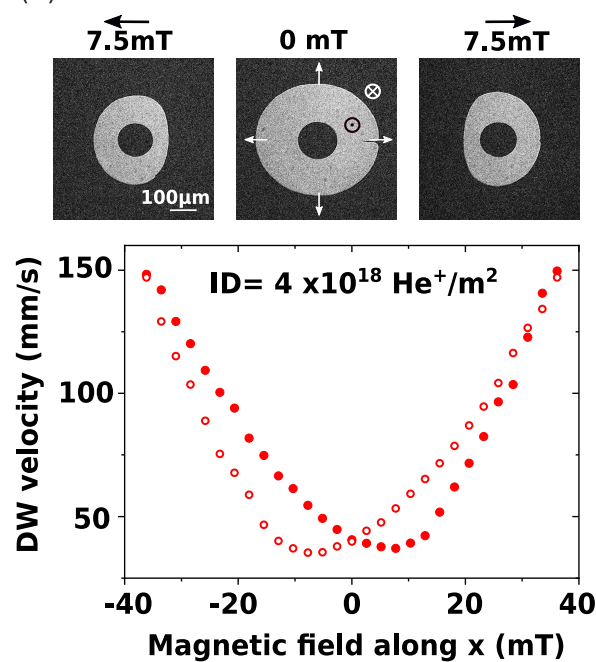

(d)
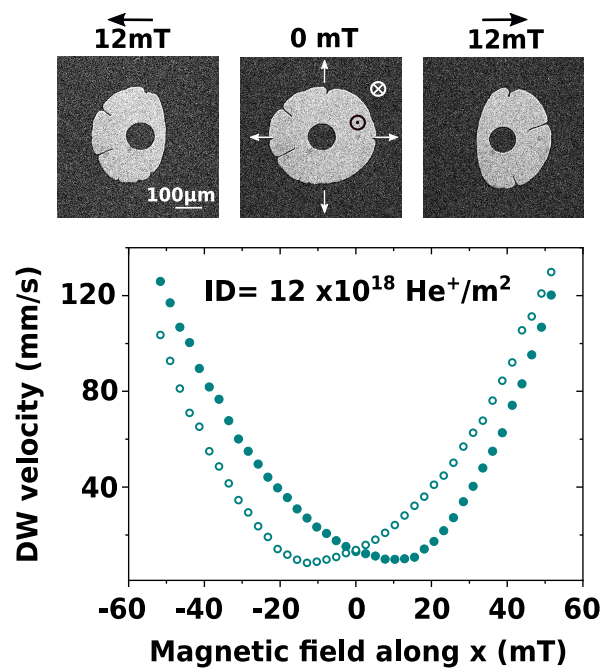

(e)
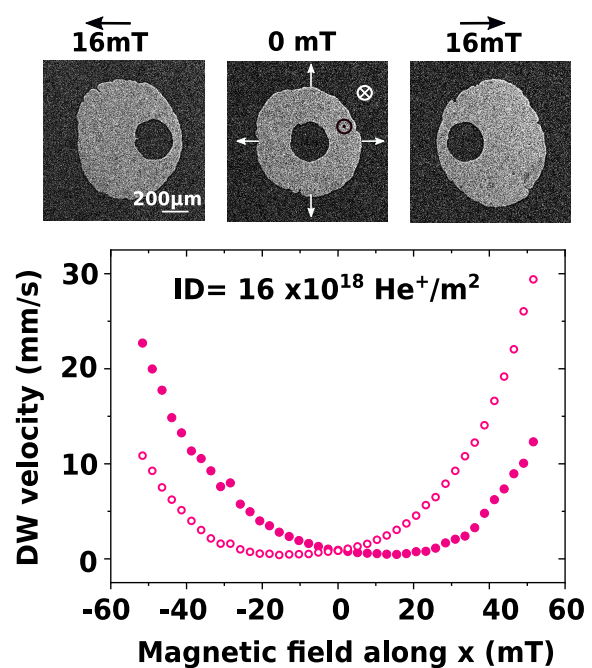

FIG. 4: Effect of irradiation induced DMI on the expansion of a bubble domain. Magneto-optical Kerr images are taken after a perpendicular field pulse of $1.9 \mathrm{mT}$ under zero and in-plane fields $\pm H_{x} \approx \pm H_{D M I}$. White arrows indicate the direction of the magnetisation inside the DW. The velocity curves for the left and right propagating DWs as a function of $H_{x}$ are also presented in (a) for the pristine film and for IDs of 4, 8, 12 and $16 \times 10^{18} \mathrm{He}^{+} / \mathrm{m}^{2}$ in (b), (c), (d) and (e), respectively. 
(a)
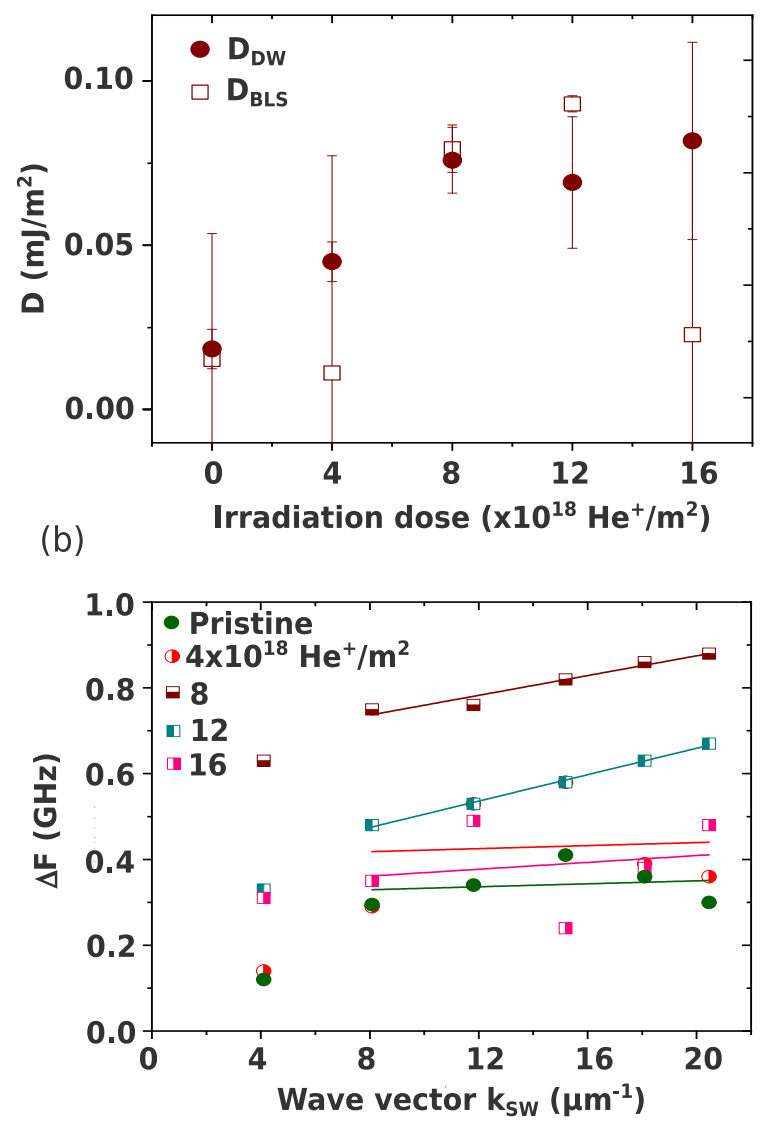

FIG. 5: (a) DMI constant $D$ as a function of the irradiation dose obtained by DW motion measurements and BLS. (b) $\Delta F$ as a function of the wave vector for pristine and irradiated films with $\mathrm{ID}=2,4,8,12$ and $16 \times 10^{18} \mathrm{He}^{+} / \mathrm{m}^{2}$. Solid lines linear fits of the high $k_{s w}$ regime from which the $D_{B L S}$ values presented in (a) were extracted.

$\mathrm{Ta} / \mathrm{CoFeB}$ and top $\mathrm{CoFeB} / \mathrm{MgO}$ interfaces increases upon irradiation and consequently can introduce an irradiation dependent modification of interface related phenomena such as DMI. This is in line with experiments conducted in $\mathrm{Pt} / \mathrm{Co} / \mathrm{Pt}$ multilayers grown at different Ar pressure, where the difference in structural quality between the bottom and top interfaces can result in an increase of the magnitude of the $\mathrm{DMI}^{18}$. Additionally, the DMI coefficient has been shown to depend on the degree of hybridisation between the $3 d$ orbitals of the ferromagnetic metal and the $5 d$ orbitals of the heavy metal $^{6}$. Therefore, irradiation assisted interdiffusion may favour alloying with the heavy metal increasing the $5 d$ band filling of the Ta and, in turn, DMI.

Recent studies presenting the effects of $\mathrm{Ar}^{+}$irradiation in $\mathrm{Pt} / \mathrm{Co} / \mathrm{Pt}$ films ${ }^{36}$ show that $H_{D M I}$ can be modulated by two main mechanisms: intermixing at the $\mathrm{Co} / \mathrm{Pt}$ interfaces and progressive removal of the top $\mathrm{Pt}$ layer. In the present case, as mentioned, the first mechanism is at play and although the energies used in this study are one order of magnitude larger than the highest used in $\mathrm{Ar}^{+}$irradiation, for the same doses no etching or large structural damages occur under irradiation with lighter $\mathrm{He}^{+}$ions. In addition, although disorder is introduced in the system, in the ID range presented here the coercive field remains fairly constant ${ }^{16}$. Given the fact that coercivity in this system is defined by domain nucleation happening at rare and relatively large defects a rather constant coercivity indicates that no new large defects are created during irradiation. On the other hand, defects that can interact with the DW not only in the creep regime but also at higher fields are introduced in the system and their role in the DW flow dynamics will be discussed in the following.

\section{THE EFFECT OF IRRADIATION IN FLOW DW DYNAMICS}

As mentioned earlier, at high fields beyond the creep and depinning regimes a region of saturation of the maximum DW velocity is observed rather than a linear dependence. In this context, the analysis of the DW dynamics can not be made simply considering a one dimensional model and therefore micromagnetic simulations were performed using the GPU-based software package MuMax $3^{37}$. A $2056 \times 1024 \times 1 \mathrm{~nm}$ computational region, discretized in $2 \times 2 \times 1 \mathrm{~nm}^{3}$ cells, was considered. A different set of values for the anisotropy constant $K_{u}$, saturation magnetization $M_{s}$, exchange constant $A$ and DMI constant $D\left(D_{D W}\right)$ were used for each ID according to the experimentally determined values that have been already introduced. The damping constant $\alpha$ was set to 0.015 , an average value observed in non-irradiated $\mathrm{Ta} / \mathrm{CoFeB} / \mathrm{MgO}$ films ${ }^{23}$. A detailed discussion on the damping parameter can be found at the end of this section. As mentioned earlier, disorder is a key aspect in the study of the effects of ion irradiation therefore it has been introduced in this model by generating a grain distribution of $10 \mathrm{~nm}$ average diameter using a Voronoi tessellation of the plane ${ }^{38}$. Both uniaxial anisotropy constant $K_{u}$ and easy axis orientation $\hat{u}$ values are assigned randomly to each grain with a normal distribution around their nominal value and a standard deviation $\sigma$. Starting from a Bloch DW in the middle of the computational region separating two antiparallel domains, the system is let to relax before the field is applied. Later, with the field on, the system is let to evolve until the average magnetization in the perpendicular direction $m_{z}$ reaches 0.8 . The domain wall velocity is computed from the change in the average normalized magnetization as $v=\frac{1}{2} L_{x} \frac{\Delta<m_{z}>}{\Delta t_{w}}$, where $\Delta t_{w}$ is the time window of the simulation. Simulations with three different grain distributions are carried out for each value of the applied field, from where the average velocity is computed.

Fig. 6 (a) shows the simulated DW velocity curves obtained using the experimental values of the intrinsic parameters and an anisotropy dispersion of $\sigma=0.06$ for the 
pristine and irradiated systems. The curves obtained reproduce well the profile of the experimental curves, however, the effect of irradiation on the intrinsic parameters does not translate into a significant variation of the maximum DW velocity as observed experimentally. As mentioned, DW velocity measurements in the creep regime have indicated that the irradiation-induced changes in the intrinsic properties of the system may be accompanied by an increase in defect density while also showing clear evidence of an irradiation induced change in composition at the $\mathrm{CoFeB} / \mathrm{MgO}$ interface ${ }^{16}$. In addition, there is a correlation between the DW velocity at the end of the creep regime (highly sensitive to defects) and the maximum DW velocity obtained well into the flow regime (Fig. 1) and evidence of a modification of the Ta/CoFeB interface width upon irradiation (Fig. 3). This leads to the conclusion that disorder plays a crucial role in irradiated systems and that it is an aspect that needs to be considered carefully when analysing DW dynamics even beyond the creep regime. In order to consider a variation of disorder as a function of ID the anisotropy dispersion $\sigma$, that simulates disorder in the system, was allowed to increase from the value of the pristine film $\left(\sigma_{0}=0.06\right)$ up to a saturation value $\left(\sigma_{\max }\right)$ as an arctangent function of the irradiation dose: $\sigma(I D)=0.06+\frac{0.11}{\pi} \arctan \left(\frac{I D}{2}\right)$ (see Fig. $6(\mathrm{c})$ ). This particular estimation was chosen to resemble the evolution of the $\mathrm{Ta} / \mathrm{CoFeB}$ interface width presented in Fig. 3 which has been taken as a reference for the increase of disorder in the system.

In this framework the simulations can reproduce the initial increase in velocity up to $\mathrm{ID}=8 \times 10^{18} \mathrm{He}^{+} / \mathrm{m}^{2}$ followed by a small subsequent decrease as shown in Fig. 6 (b). The good correspondence between the simulations and the experimental results reveals the existence of a maximum level of disorder at around $\mathrm{ID}=8 \times 10^{18}$ $\mathrm{He}^{+} / \mathrm{m}^{2}$ that will not be significantly incremented by using higher irradiation doses, in contrast to the effects of irradiation on anisotropy and saturation magnetisation. This is also the profile of the changes observed in DMI as a function of ID, therefore linking the evolution of disorder to the strengthening of DMI and the changes induced at the $\mathrm{Ta} / \mathrm{CoFeB}$ interface.

As seen in Fig. 6 (a), considering solely the irradiation induced increase of DMI is not enough to explain the observed increase in DW velocity. This feature is most likely due to the low values of DMI present in the system before irradiation, a known feature for $\mathrm{CoFeB}$ systems grown on a Ta buffer layer ${ }^{8}$. This scenario might be different in systems with large DMI where this parameter is the determining factor for DW velocities in the flow regime ${ }^{7}$. In the present case, the increase in DW velocity seems to be directly related to irradiation induced disorder, beyond the changes that this produces in the intrinsic parameters. Previous theoretical studies show that the spin precession inside a DW in a perfect $\mathrm{CoFeB}$ system constitutes the main energy dissipation mechanism ${ }^{39}$. However, in disordered films the pinning/depinning of DWs at grain boundaries, where a distribution of anisotropy is (a)

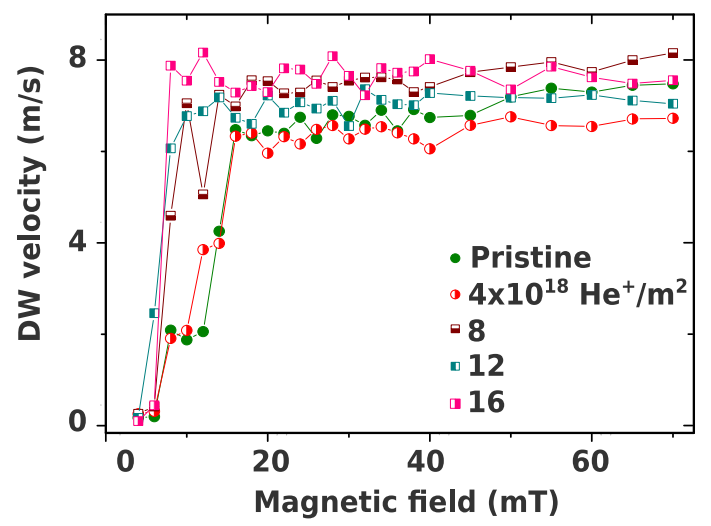

(b)

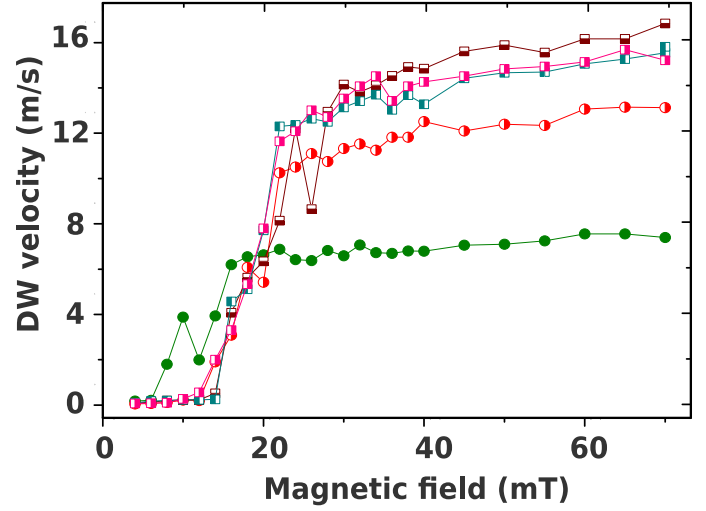

(c)

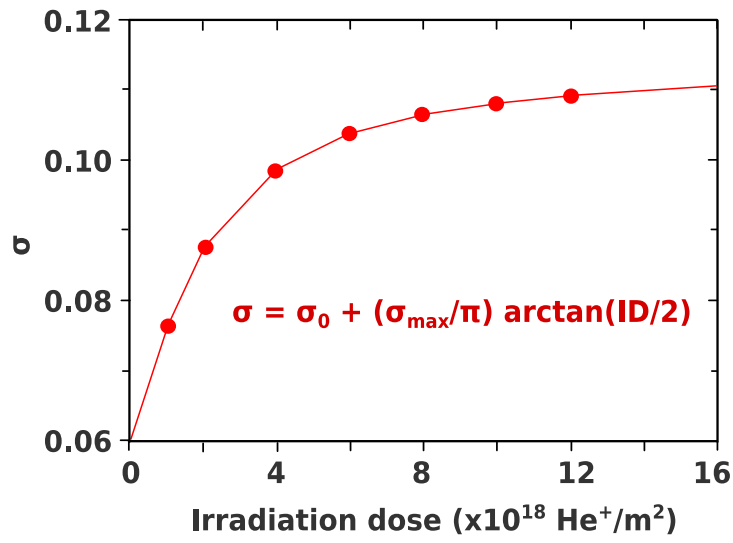

FIG. 6: Micromagnetic simulations considering the irradiation induced changes in the intrinsic parameters with (a) a constant disorder and (b) an ID dependent disorder. The simulated disorder variation with ID is plotted in (c).

introduced, triggers spin wave emission. This additional energy dissipation channel can be interpreted as an extrinsic effective damping and leads to a faster relaxation of the system and consequently a higher DW velocity, which is not observed in simulations of films without disorder. This contribution scales with the defect density, in this case with irradiation dose, and favours DW motion 
(a)

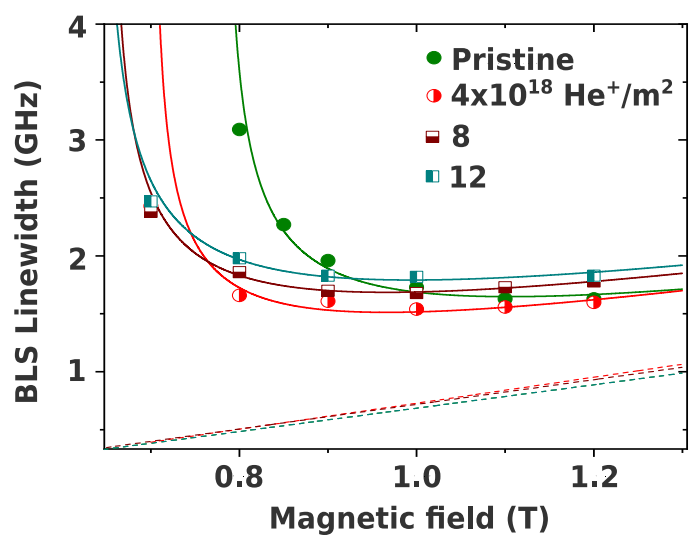

(b)

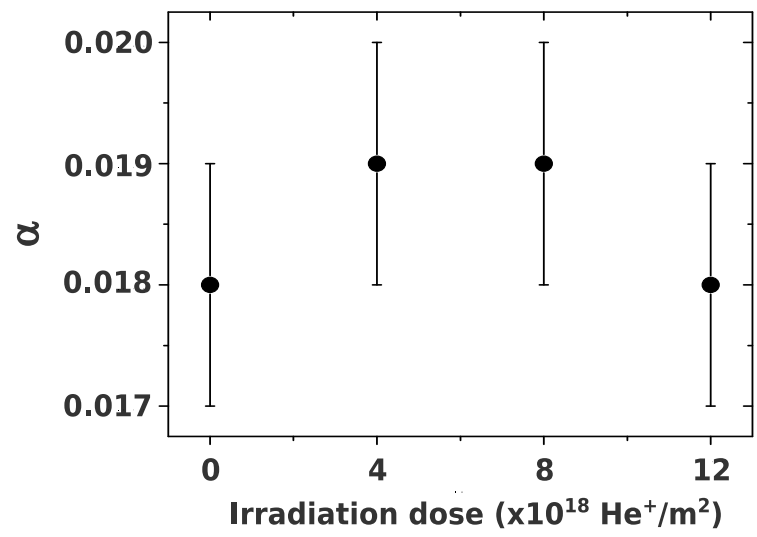

FIG. 7: BLS linewidth as a function of the applied in-plane magnetic field, solid lines going through the datapoints correspond to a fitting using Eq. (2) while the lines at the bottom of the graph are simulated neglecting the inhomogeneous contribution to the effective damping. (b) Gilbert damping parameter extracted from the fittings in (a).

in the flow regime ${ }^{39}$. This, once again, indicates that the observed irradiation induced DMI is not the determining factor and that disorder is responsible for the observed increase in DW velocity in the flow regime.

In these simulations the value of the Gilbert damping $(\alpha)$ parameter was kept constant, the value chosen being the one that corresponds to a similar pristine film of the same composition $^{23}$. Previous studies have shown that during irradiation-assisted crystallization of $\mathrm{CoFeB} / \mathrm{MgO}$ films the effective damping obtained by ferromagnetic resonance, including the Gilbert damping and an inhomogeneous broadening due to disorder, tends to increase starting from irradiation doses close to ID $=1.5 \times 10^{19} \mathrm{He}^{+} / \mathrm{m}^{2}$ but that below that threshold, that are the values concerned in this study, no significant changes occur ${ }^{20}$. Nevertheless, the contribution from the Gilbert damping to the global effective damping in this system is expected to be considerably reduced due to the key role played by defects that can significantly increase the inhomoge- neous contribution. Fig. 7 (a) shows the BLS linewidth as a function of the applied in-plane magnetic field where the profile of the datapoints is indeed far from the linear dependence that is expected when Gilbert damping is dominating. Therefore, a model considering an inhomogeneous contribution in the form of a variation of the anisotropy field across the sample $\Delta H_{K}$ has been used, in line with the anisotropy distributions that have been introduced in the micromagnetic simulations. In this model the BLS linewidth $\Delta \omega$ depends on the applied magnetic field $H>H_{K}$ as follows ${ }^{40}$ :

$$
\Delta \omega=\alpha \gamma\left(2 H-H_{K}\right)+\frac{\gamma H \Delta H_{K}}{2 \sqrt{H^{2}-H H_{K}}} .
$$

The first term of this expression contains the intrinsic damping characterised by the Gilbert damping and the second term describes the inhomogeneous broadening due to variations in the anisotropy field. Solid lines going through the datapoints in Fig. 7 (a) are the result of a fitting using this full expression while the lines in the bottom part of the graph are done using the same fitting parameters but only considering the first term of this expression. These curves are the expected result in the absence of a significant contribution from $\Delta H_{K}$, which shows that the inhomogeneous component is entirely dominating the observed BLS linewidth. Using this expression the values of the Gilbert damping were extracted and are plotted in Fig. 7 (b), which does not show to be greatly affected by irradiation and also shows values close to the estimated value of 0.015 previously reported. The second fitting parameter that was obtained was $\Delta H_{K}$ which presented a monotonous increase going from $4.5 \%$ of the anisotropy field for the non irrradiated film to up to $7.5 \%$ for $\mathrm{ID}=1.2 \times 10^{19} \mathrm{He}^{+} / \mathrm{m}^{2}$. These values are also in the range of the estimations made by micromagnetic simulations and further validate the analysis that was previously presented.

\section{CONCLUSION}

In conclusion, this study presents the influence of $\mathrm{He}^{+}$irradiation on the interfacial structure and the high field DW dynamics beyond the creep regime in $\mathrm{Ta} / \mathrm{CoFeB} / \mathrm{MgO}$ films. These results indicate that in films with a nearly quenched DMI $\mathrm{He}^{+}$irradiation induces a broadening of the $\mathrm{Ta} / \mathrm{CoFeB}$ interface that leads to an increase in the magnitude of the DMI, evidenced by DW motion measurements and BLS. We studied the DW dynamics in the flow regime supported by micromagnetic simulations that indicate the crucial role played by the irradiation induced disorder beyond the changes produced in the intrinsic parameters like magnetic anisotropy, DMI and saturation magnetisation. Disorder also seems to be the key aspect responsible for the increase in DW velocity upon irradiation and it reveals itself as the dominant feature in the BLS linewidth while the value of 
the Gilbert damping is not greatly affected. This thorough study of the effects of ion irradiation on ultra thin films with perpendicular anisotropy provides insight for the understanding of important physical phenomena like the enhancement of intefacial DMI and the role of defects in DW motion at high fields. In addition, it is also relevant for the development of spintronics devices for applications where magnetic patterning of DMI and DW velocity can be of considerable interest.

\section{Acknowledgments}

We gratefully acknowledge financial support from the European Union FP7 Program through contracts MAG-
WIRE No.257707 and ITN WALL No. 608031 as well as from the French National Research Agency though the projects COMAG and ELECSPIN. We also want to acknowledge funding from the project CNRS PREMAT 'Spin-Ion' and from the Conseil Regional dle-de-France through the DIM NanoK (BIDUL) project.
* Electronic address: liza.herrera-diez@c2n.upsaclay. fr

1 I. Dzyaloshinsky, Journal of Physics and Chemistry of Solids 4, 241 (1958).

2 T. Moriya, Physical Review 120, 91 (1960).

3 Hongxin Yang, André Thiaville, Stanislas Rohart, Albert Fert and Mairbek Chshiev, Phys. Rev. Lett. 115, 267210 (2015).

4 A. Thiaville, S. Rohart, E. Jué, V. Cros and A. Fert, EPL 100, 57002 (2012).

5 Jacob Torrejon, Junyeon Kim, Jaivardhan Sinha, Seiji Mitani, Masamitsu Hayashi, Michihiko Yamanouchi and Hideo Ohno, Nat. Comm. 5, 4655 (2014).

${ }^{6}$ X. Ma, G. Yu, C. Tang, X. Li, C. He, J. Shi, K. L. Wang, and X. Li, Physical Review Letters 120, 157204 (2018).

7 T. Ha Pham, J. Vogel, J. Sampaio, M. Vanatka, J.-C. Rojas, M. Bonfim, D.S. Chaves, F. Choueikani, P. Ohresser, E. Otero, A. Thiaville and S. Pizzini,Europhys. Lett. 113, 67001 (2016).

8 J.-P. Tetienne, T. Hingant, L.J. Martínez, S. Rohart, A. Thiaville, L. Herrera Diez, K Garcia, J.-P. Adam, J.-V. Kim, J.-F. Roch, I.M. Miron, G. Gaudin, L. Vila, B. Ocker, D. Ravelosona and V. Jacques, Nature Comm. 6, 6733 (2015).

9 A. Fert, N. Reyren, and V. Cros, Nature Reviews Materials 2, 17031 (2017).

10 S. Woo, K. Litzius, B. Krger, M.-Y. Im, L. Caretta, K. Richter, M. Mann, A. Krone, R. M. Reeve, M. Weigand, P. Agrawal, I. Lemesh, M.-A. Mawass, P. Fischer, M. Kläui, and G. S. D. Beach, Nature Materials 15, 501 (2016).

11 G. Yu, P. Upadhyaya, X. Li, W. Li, S. K. Kim, Y. Fan, K. L. Wong, Y. Tserkovnyak, P. K. Amiri, and K. L. Wang, Nano Letters 16, 1981 (2016).

12 P.J. Metaxas, J.P. Jamet, A. Mougin, M. Cormier, J. Ferré, V. Baltz, B. Rodmacq, B. Dieny and R.L. Stamps, Phys. Rev. Lett. 99, 217208 (2007).

13 V. Jeudy, A. Mougin, S. Bustingorry, W. Savero Torres, J. Gorchon, A. B. Kolton, A. Lematre, and J.-P. Jamet, Phys. Rev. Lett. 117, 057201 (2016).

14 S. Lemerle, J. Ferré, C. Chappert, V. Mathet, T. Giamarchi, P. Le Doussal, Phys. Rev. Lett. 80, 849 (1998).

15 L. Herrera Diez, V. Jeudy, G. Durin, A. Casiraghi, Y.T. Liu, M. Voto, G. Agnus, D. Bouville, L. Vila, J. Langer,
B. Ocker, L. Lopez-Diaz, and D. Ravelosona, Phys. Rev. B 98, 054417 (2018).

${ }^{16}$ L. Herrera Diez, F. García-Sánchez, J.-P. Adam, T. Devolder, S. Eimer, M. S. El Hadri, A. Lamperti, R. Mantovan, B. Ocker, and D. Ravelosona, Appl. Phys. Lett. 107, 032401 (2015).

17 A. Hrabec, N. A. Porter, A. Wells, M. J. Benitez, G. Burnell, S. McVitie, D. McGrouther, T. A. Moore, and C. H. Marrows, Phys. Rev. B 90, (2014).

18 A. W. J. Wells, P. M. Shepley, C. H. Marrows, and T. A. Moore, Phys. Rev. B 95, (2017).

19 B. Zimmermann, W. Legrand, D. Maccariello, N. Reyren, V. Cros, S. Blgel, and A. Fert, Appl. Phys. Lett. 113, 232403 (2018).

20 T. Devolder, I. Barisic, S. Eimer, K. Garcia, J.-P. Adam, B. Ockert and D. Ravelosona, J. Appl. Phys. 113, 203912 (2013).

21 R. Diaz Pardo, W. Savero Torres, A. B. Kolton, S. Bustingorry, and V. Jeudy. Phys. Rev. B 95, 184434 (2017).

22 K. Yamada, J.-P. Jamet, Y. Nakatani, A. Mougin, A. Thiaville, T. Ono and J. Ferré, Appl. Phys. Ex. 4, 113001 (2011).

${ }^{23}$ C. Burrowes, N. Vernier, J.-P. Adam, L. Herrera Diez, K. Garcia, I. Barisic, G. Agnus, S. Eimer, J.-V. Kim, T. Devolder, A. Lamperti, R. Mantovan, B. Ockert, E. E. Fullerton, and D. Ravelosona, Appl. Phys. Lett. 103, 182401 (2013).

24 L. Lutterotti, D. Chateigner, S. Ferrari, and J. Ricote, Thin Solid Films 450, 34 (2004).

25 Soong-Geun Je, Duck-Ho Kim, Sang-Cheol Yoo, ByoungChul Min, Kyung-Jin Lee and Sug-Bong Choe, Phys. Rev. B 88, 214401 (2013).

26 R. Soucaille, M. Belmeguenai, J. Torrejon, J.-V. Kim, T. Devolder, Y. Roussigné, S.-M. Chérif, A. A. Stashkevich, M. Hayashi and J.-P. Adam, Phys. Rev. B 94, 104431 (2016).

27 M. Yamanouchi, A. Jander and P. Dhagat, IEEE Magn. Lett. 2, 3000304 (2011).

28 G. Asti, M. Ghidini, M. Mulazzi, R. Pellicelli, M. Solzi, K. Chesnel, A. Marty, Phys. Rev. B 76, 094414 (2007).

29 A. Hubert and R. Schäfer Magnetic Domains SpringerVerlag, Berlin, p. 390, (1998).

30 A proportionality factor is extracted from the non irradi- 
ated film value of $2.3 \times 10^{-11} \mathrm{~J} / \mathrm{m}$ by considering the corresponding value of $M_{s}$.

31 M. Belmeguenai, D. Apalkov, Y. Roussigné, S. M. Cherif, A. Stashkevich, G. Feng and X. Tang, J. Phys. D: Appl. Phys. 50, 415003 (2017).

32 M. Belmeguenai, J.-P. Adam, Y. Roussigné, S. Eimer, T. Devolder, J.-V. Kim, S. M. Cherif, A. Stashkevich and A. Thiaville, Phys. Rev. B 91, 180405(R) (2015).

${ }^{33}$ H. Bernas, J.-P. Attané, K.-H. Heinig, D. Halley, D. Ravelosona, A. Marty, P. Auric, C. Chappert, and Y. Samson, Phys. Rev. Lett. 91, 077203 (2003).

34 J.Ziegler, J. Biersak and U.Littmark, The stopping of ions in matter Pergamon, New York, (1985).

35 M. Cecot, L. Karwacki, W. Skowronski, J. Kanak, J. Wrona, A. Zywczak, L. Yao, S. van Dijken, J. Barnas, and
T. Stobiecki, Scientific Reports 7, 968 (2017).

36 A. L. Balk, K-W. Kim, D. T. Pierce, M. D. Stiles, J. Unguris and S. M. Stavis, Phys. Rev. Lett. 119, 077205 (2017).

37 A. Vansteenkiste, J. Leliaert, M. Dvornik, M. Helsen, F. Garcia-Sanchez and B. Van Waeyenberge, AIP Adv. 4, 107133 (2014).

38 J. Leliaert, B. Van de Wiele, A. Vansteenkiste, L. Laurson, G. Durin, L. Dupré, B. Van Waeyenberge. J. Appl. Phys. 115, 233903 (2014).

39 M. Voto, L. Lopez-Diaz and L. Torres, J. Phys. D: Appl. Phys. 49, 185001 (2016)

40 A. Capua, S.-H. Yang, T. Phung, and S. S. P. Parkin, Phys. Rev. B 92, 224402 (2015). 